\title{
Dynamic Pressure of Seabed around Buried Pipelines in Shallow Water
}

\author{
Changjing Fu, ${ }^{1,2}$ Guoying Li, ${ }^{1}$ Tianlong Zhao, ${ }^{1,2}$ and Donghai Guan ${ }^{3}$ \\ ${ }^{1}$ Nanjing Hydraulic Research Institute, Nanjing 210029, China \\ ${ }^{2}$ Chongqing Jiaotong University, Chongqing 400074, China \\ ${ }^{3}$ Nanjing University of Aeronautics and Astronautics, Nanjing 210016, China \\ Correspondence should be addressed to Guoying Li; gyli@nhri.cn
}

Received 27 April 2015; Revised 3 August 2015; Accepted 1 September 2015

Academic Editor: Manuel Pastor

Copyright (C) 2015 Changjing Fu et al. This is an open access article distributed under the Creative Commons Attribution License, which permits unrestricted use, distribution, and reproduction in any medium, provided the original work is properly cited.

Due to the obvious nonlinear effect caused by the shallow waves, the nonlinear wave loads have a great influence on the buried pipelines in shallow water. In order to ensure their stability, the forces on the pipelines that resulted from nonlinear waves should be considered thoroughly. Based on the Biot consolidation theory and the first-order approximate cnoidal wave theory, analytical solutions of the pore water pressure around the buried pipelines in shallow water caused by waves are firstly derived in this paper. The dynamic response of the seabed around the pipelines under the action of irregular waves is explored in laboratory, and the results are compared with the analytical ones. Experiments show that the maximum value of the pore water pressure is at the upper part of the pipelines, and the value at the bottom of the pipelines is the minimum. The pore water pressure amplitude at the upper half of the seabed that arises from waves has a larger fluctuation, and the amplitude at the lower half remains stable. For the sandy seabed, the pore water pressure at the same depth changes over time, with the fluctuating amplitude, and the theoretical values meet well with the test results.

\section{Introduction}

Submarine pipeline installation is one of the key projects in exploitation activities of ocean oil and gas resources. The submarine pipeline, called the "lifeline" of the marine oil and gas fields, has become the energy transport artery gradually with the quickening pace of exploration and exploitation of offshore oil. The layout modes of the submarine pipelines can be mainly divided into two types: embedment (deep and shallow) and direct placement. Due to the high costs and difficult construction of deep buried pipelines and the vulnerability to the external loads for the direct placement of the pipelines, the shallow buried mode is mostly adopted in the construction process of the pipelines. Generally, the marine projects in shallow water are greatly influenced by wave loads.

The wave pressure on the seabed surface has a periodical change with the wave propagation, and it will cause excess pore water pressure and effective stress while it transfers to the seabed. Thus, it changes the distribution of the effective stress on the seabed. For evaluating the stability of submarine pipelines under wave action rationally and therefore ensuring their normal operation, the effect of the pore water pressure of seabed around the pipelines induced by waves should be considered. On the basis of the previous research findings and considering the compressibility of the pore water and the seabed, the analytic solutions of the pore water pressure around the buried pipelines under the nonlinear wave loads are derived by the method of separation of variables based on the Biot consolidation and the first-order approximate cnoidal wave theory. The laboratory tests are performed to verify the theory.

\section{Background}

The dynamic response of marine soils has received much attention since the 1940s. The early theories are based on 
the assumptions of rigid soil skeleton and incompressible pore fluid and it is also supposed that the flow of pore water meets Darcy's law and the distribution of the excess pore water pressure satisfies the Laplace formulation [15]. The subsequent analytical approaches assume that the pore water is compressible and the seabed is not deformable [6-8]. The third-stage approaches took the compressibility of pore water into consideration and assumed that the flow of pore water met Darcy's law. With the Biot theory, these approaches suppose that the skeleton of marine soils is isotropic elastic body, which meant that the mechanical properties of the seabed under small deformation are reversible, linear, and isotropic and the flow of pore water meets Darcy's law. Considering the stress-strain relation and movement of pore water and soil skeleton, the dynamic response of elastic marine soils with finite or infinite depth under the effect of linear progressive waves was studied by using close-form analytical approaches. Many researchers [912] adopted this kind of approaches and achieved various findings.

When the pipeline is buried in the seabed, the interaction between pipelines, soils, and waves should be considered. As a consequence, the problem of fluent flows in porous permeable seabed and distribution of the excess pore water pressure becomes more complicated. Earlier, the researchers fail to consider the compression of pore water and the deformation of soil skeleton. The analytic solutions of seepage pressure around the buried pipelines in marine soils with finite or infinite depth were derived on the assumption that the soils are a rigid body based on the potential flow theory [13-15]. Under the assumption of permeable rigid seabed, some researchers studied the distribution of the excess pore water pressure around the buried pipelines in the seabed by using the finite difference, finite element, and boundary integral methods [16-18]. Then, by using the Biot consolidation theory, some scholars analyzed the distribution of the excess pore water pressure around the buried pipelines under the action of waves [19-27].

The theories mentioned above adopt the linear wave theory. The nonlinearity of shallow water waves is obvious and should be considered thoroughly. Recently, scholars mostly used the Biot consolidation theory, finite element method, and Stokes wave theory to study the dynamic response of seabed and pipelines [28-30]. For the application of wave theories, Le Méhauté [31] deemed that the Stokes wave theory is applicable for the deep water. Besides, the solitary wave theory and cnoidal wave theory were more suitable for the shallow water [32]. In recent years, many scholars began to be concerned about the interaction between solitary waves and offshore structures [33-35]. Based on the solitary wave diffraction theory and the Biot wave seepage consolidation theory, Li et al. [35] deduced the mathematical solution of the seepage pressure field in seabed through the eigenfunction method, and they also evaluated the seepage loads at the bottom of vertical cylinder resting on porous seabed. Xiao and Huang [36] established three-dimensional numerical model and analyzed the interaction between solitary waves and cylinder piles in a coastal surf zone. Some conclusions in the theory of solitary waves are different from the natural

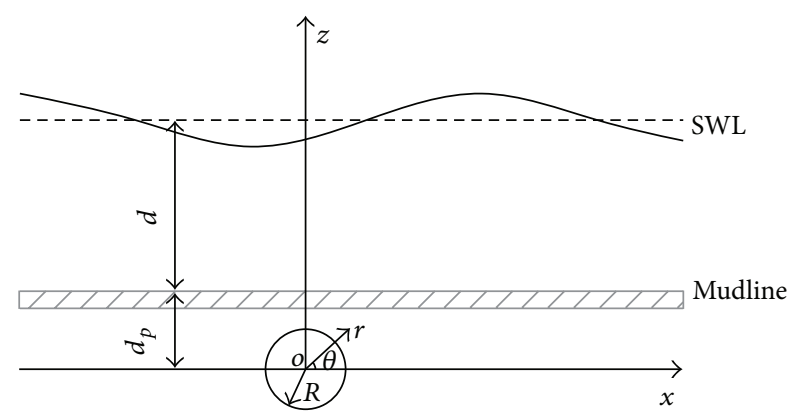

FIgURE 1: Definition sketch.

conditions. Therefore, in the study of nonlinear influence of shallow water waves, the cnoidal wave theory is more appropriate. Based on the first-order approximation cnoidal wave theory, Sun and Qiu [37] derived the analytical solution of wave forces on the buried pipelines under the action of nonlinear water waves in shallow water by using the image method. Based on the cnoidal wave theory, Xu et al. [38] and Zhou et al. [39] calculated wave pressure on seabed surface under the effect of waves in the littoral region and also studied the response of pore pressure on the seabed with the action of cnoidal waves by analyzing parameters. According to their conclusion, it was found that the permeability of seabed and compressibility of pore water had great impact on the response of pore pressure on the seabed.

\section{Governing Equations and Boundary Conditions}

As shown in Figure 1, it is assumed that the seabed is infinitely deep, and the pipeline with radius of $R$ is buried in the seabed, with buried depth of $d_{p}$. The waves propagate along the perpendicular direction to the pipeline, that is, $x$-axis, and the direction of $z$-axis is vertically upward. Based on the Biot consolidation theory, the governing equations for the soils can be expressed as [40]

$$
\begin{aligned}
-G \nabla^{2} w_{x}+\frac{G}{1-2 \nu} \cdot \frac{\partial}{\partial x} \varepsilon_{V}+\frac{\partial p}{\partial x} & =0 \\
-G \nabla^{2} w_{z}+\frac{G}{1-2 \nu} \cdot \frac{\partial}{\partial z} \varepsilon_{V}+\frac{\partial p}{\partial z} & =-\gamma \\
\frac{\partial \varepsilon_{V}}{\partial t}+\frac{k_{s}}{\gamma_{w}} \nabla^{2} p & =0
\end{aligned}
$$

where $G$ is the shear modulus of soils; $\nabla^{2}$ denotes the two-dimensional Laplace operator; $w_{x}$ stands for the soil displacement in $x$ direction; $v$ is Poisson's ratio of soils; $\varepsilon_{V}$ stands for $-\left(\partial w_{x} / \partial x+\partial w_{z} / \partial z\right)$, the volumetric strain; $p$ is pore water pressure; $x$ denotes the horizontal coordinate axis as defined in Figure $1 ; w_{z}$ is the soil displacement in $z$ direction; $z$ is the depth into the soils as defined in Figure 1; $k_{s}$ is the coefficient of permeability; $\gamma_{w}$ is the weight density of sea water.

In order to simplify analysis of the dynamic response around the submarine pipelines under the action of waves 
by using the Biot consolidation theory, the following assumptions are adopted.

(1) The seabed soils are isotropic and homogeneous. (2) The compression coefficient of seabed soil skeleton and the pore water is constant. (3) The deformation of the seabed soil skeleton obeys the Hooke law. (4) The pore water is cohesionless fluid. (5) The aquifer above the seabed is incompressible. (6) The wave field above the seabed is irrotational. (7) Darcy's law is satisfied in the seepage of seabed. (8) The coefficient of permeability of seabed soils is constant. (9) The materials of the pipe wall are impermeable. (10) The energy loss in the propagation of waves is neglected. The seabed movement induced by waves is micro oscillation under hydrostatic equilibrium state, so the above assumptions are reasonable.

For the compressible fluid in compressible medium, the governing equation for motion can be written as [41]

$$
\frac{k_{s}}{\gamma_{w}} \nabla^{2} p=\frac{n_{s}}{K} \frac{\partial p}{\partial t}+\frac{\partial \varepsilon_{V}}{\partial t}
$$

where $n_{s}$ is the porosity and $K$ is the bulk modulus of water $\left(K=2.18 \times 10^{9} \mathrm{~Pa}\right)$.

It is difficult to solve the coupled equations (1) and (2), so the displacement term is eliminated, and the governing equation for the pore water pressure of $p$ can be written as [42]

$$
\nabla^{2} p=C_{s} \frac{\partial p}{\partial t}
$$

in which $C_{s}=\left(\gamma_{w} / k_{s}\right)\left(n_{s} / K+(1 / 2 G)((1-2 \nu) /(1-\nu))\right)$.

It is supposed that the seabed is infinitely deep and the soils are homogeneous medium and $p$ satisfies the following boundary conditions:

$$
\begin{aligned}
p & =p_{w}, \\
z & =d_{p}, \\
\frac{\partial p}{\partial r} & =0, \\
r & =R, \\
p & =0, \\
z & \longrightarrow-\infty,
\end{aligned}
$$

where $R$ is the radius of the pipe; $d_{p}$ is the buried depth to the center line of the pipe; $r=\left(x^{2}+z^{2}\right)^{1 / 2}$ as defined in Figure 1.

In the calculation of the excess pore water pressure of the seabed surface, the viscous and frictional forces of water are always ignored, the vertical effective stress and shear stress in the surface of seabed are not considered, and it is approximately equal to the wave pressure at the bottom of seabed caused by waves, which is [37]

$$
\begin{aligned}
p_{w}= & \rho_{w} \frac{H}{d} \frac{\sqrt{g d}}{k} \sum_{n=1}^{\infty} n \omega \frac{2 \pi^{2}}{\kappa^{2} F^{2}(\kappa)} \frac{\varepsilon^{n}}{1-\varepsilon^{2 n}} \\
& \cdot \exp [i n(k x-\omega t)],
\end{aligned}
$$

where $\rho_{w}$ is the seawater density; $H$ is the wave height; $d$ is the still water depth; $g$ is the gravitational acceleration; $k$ is the wave number determined in accordance with the linear wave theory dispersion equation; $\omega$ is the frequency of radian wave; $\kappa$ is modulus; $F(\kappa)$ is the first kind of ellipse integral; and $\varepsilon=$ $\exp \left(-\pi F^{\prime}(\kappa) / F(\kappa)\right)$, in which $F^{\prime}(\kappa)$ is the first kind of ellipse integral; $i=\sqrt{-1}$ and $t$ stands for time. In addition, equation above was proposed based on first-order approximate cnoidal wave theory.

\section{Solutions}

Because of the existence of the pipeline, the fluid in the seabed will scatter when it reaches the pipeline. Therefore, $p$ can be divided into two parts, one is the seepage pressure caused by waves, denoted by $p_{1}$, and the other is $p_{2}$, the perturbation pressure induce by the pipe:

$$
p=p_{1}+p_{2}
$$

When there is no pipeline in the seabed, the seepage pressure $p_{1}$ caused by waves meets the following governing equations and the corresponding boundary conditions

$$
\begin{aligned}
\frac{\partial^{2} p_{1}}{\partial x^{2}}+\frac{\partial^{2} p_{1}}{\partial z^{2}} & =C_{s} \frac{\partial p_{1}}{\partial t}, \\
p_{1} & =p_{w}, \\
z & =d_{p}, \\
p_{1} & =0, \\
z & \longrightarrow-\infty .
\end{aligned}
$$

According to the boundary conditions, the seepage pressure $p_{1}$ can be easily calculated by

$$
\begin{aligned}
p_{1}= & \rho_{w} \frac{H}{d} \frac{\sqrt{g d}}{k} \sum_{n=1}^{\infty} n \omega \frac{2 \pi^{2}}{\kappa^{2} F^{2}(\kappa)} \frac{\varepsilon^{n}}{1-\varepsilon^{2 n}} \\
& \cdot \exp \left(C\left(z-d_{p}\right)+i n(k x-\omega t)\right),
\end{aligned}
$$

in which $C=\left((n k)^{2}-i n \omega C_{s}\right)^{1 / 2}$. 
Usually, the pipe is a circular tube so that the coordinates of governing equations are transformed and then the governing equations and boundary conditions of $p_{2}$ are given as follows:

$$
\begin{aligned}
\frac{\partial^{2} p_{2}}{\partial r^{2}}+\frac{1}{r} \frac{\partial p_{2}}{\partial r}+\frac{1}{r^{2}} \frac{\partial^{2} p_{2}}{\partial \theta^{2}} & =C_{s} \frac{\partial p_{2}}{\partial t}, \\
p_{2} & =0, \\
r & =d_{p}, \\
\theta & =\frac{\pi}{2}, \\
\frac{\partial p_{2}}{\partial r} & =-\frac{\partial p_{1}}{\partial r}, \\
r & =R, \\
p_{2} & =0, \\
r & \longrightarrow-\infty .
\end{aligned}
$$

By using the method of separation of variables, the perturbation pressure $p_{2}$ caused by the pipeline can be expressed as

$$
\begin{aligned}
p_{2}(r, \theta, t)= & A \sum_{m=0}^{\infty} \sum_{n=1}^{\infty} J_{m}\left(\frac{\mu_{n}^{(m)}}{d_{p}} r\right) \\
& \cdot \exp \left[-\frac{1}{C_{s}}\left(\frac{\mu_{n}^{(m)}}{d_{p}}\right)^{2} t\right] \cos (m \theta),
\end{aligned}
$$

in which $J_{m}(\cdot)$ is the first kind of Bessel function and $\mu_{n}^{(m)}$ is the positive zero of $J_{m}(\cdot)$.

According to the boundary condition $\partial p_{2} / \partial r=-\partial p_{1} / \partial r$, $r=R$, the undetermined coefficient $A$ in (10) can be derived by

$$
A=-\frac{\rho_{w}}{\exp \left(C d_{p}\right)} \frac{H}{d} \frac{\sqrt{g d}}{k} \sum_{m=0}^{\infty} \sum_{n=1}^{\infty} n \omega \frac{2 \pi^{2}}{\kappa^{2} F^{2}(\kappa)} \frac{\varepsilon^{n}}{1-\varepsilon^{2 n}} \frac{f^{\prime}(t) \exp \left[\left(1 / C_{s}\right)\left(\mu_{n}^{(m)} / d_{p}\right)^{2} t\right] \int_{0}^{2 \pi} f^{\prime}(\theta) \cos (m \theta) d \theta}{\left[\left(\mu_{n}^{(m)} / d_{p}\right) J_{m-1}\left(\left(\mu_{n}^{(m)} / d_{p}\right) R\right)-(m / R) J_{m}\left(\left(\mu_{n}^{(m)} / d_{p}\right) R\right)\right]},
$$

in which $f^{\prime}(\theta)=(C \sin \theta+i n k \cos \theta) \exp (C R \sin \theta+$ inkR $\cos \theta), f^{\prime}(t)=\exp (-i n \omega t)$.
Then the perturbation pressure $p_{2}$ induced by the pipe is calculated by

$$
p_{2}=-\frac{\rho_{w}}{\exp \left(C d_{p}\right)} \frac{H}{d} \frac{\sqrt{g d}}{m} \sum_{m=0}^{\infty} \sum_{n=1}^{\infty} n \omega \frac{\pi^{2}}{\kappa^{2} F^{2}(\kappa)} \frac{2 \varepsilon^{n}}{1-\varepsilon^{2 n}} \frac{J_{m}\left(\left(\mu_{n}^{(m)} / d_{p}\right) r\right) \cos (m \theta) f^{\prime}(t) \int_{0}^{2 \pi} f^{\prime}(\theta) \cos (m \theta) d \theta}{\left[\left(\mu_{n}^{(m)} / d_{p}\right) J_{m-1}\left(\left(\mu_{n}^{(m)} / d_{p}\right) R\right)-(m / R) J_{m}\left(\left(\mu_{n}^{(m)} / d_{p}\right) R\right)\right]} .
$$

Therefore, the seepage pressure $p$ at any point in the seabed can be described as

$$
\begin{aligned}
p= & p_{1}+p_{2} \\
= & \rho_{w} \frac{H}{d} \frac{\sqrt{g d}}{k} \sum_{n=1}^{\infty} n \omega \frac{2 \pi^{2}}{\kappa^{2} F^{2}(\kappa)} \frac{\varepsilon^{n}}{1-\varepsilon^{2 n}} \exp \left[C\left(z-d_{p}\right)+i n(k x-\omega t)\right] \\
& -\frac{\rho_{w}}{\exp \left(C d_{p}\right)} \frac{H}{d} \frac{\sqrt{g d}}{k} \sum_{m=0}^{\infty} \sum_{n=1}^{\infty} n \omega \frac{2 \pi^{2}}{\kappa^{2} F^{2}(\kappa)} \frac{\varepsilon^{n}}{1-\varepsilon^{2 n}} \frac{J_{m}\left(\left(\mu_{n}^{(m)} / d_{p}\right) r\right) \cos (m \theta) f^{\prime}(t) \int_{0}^{2 \pi} \cos (m \theta) \cdot f^{\prime}(\theta) d \theta}{\left[\left(\mu_{n}^{(m)} / d_{p}\right) J_{m-1}\left(\left(\mu_{n}^{(m)} / d_{p}\right) R\right)-(k / R) J_{m}\left(\left(\mu_{n}^{(m)} / d_{p}\right) R\right)\right]} .
\end{aligned}
$$

\section{Laboratory Tests}

Till now, the experimental studies on the wave forces on submarine pipelines under the action of waves by using the cnoidal wave theory are rare. In order to verify the accuracy of the theory in this paper, large wave flume tests are carried out, and the dynamic response of seabed around the buried pipelines in shallow water is highlighted.

5.1. Experimental Apparatus. The tests are conducted in Test Hall for Basic Sediment Law of Nanjing Hydraulic Research 


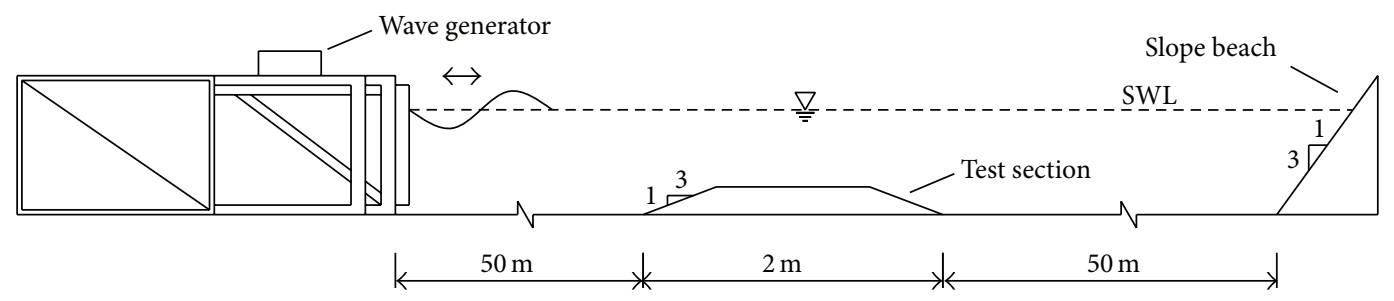

FIGURE 2: Schematic of wave flume.

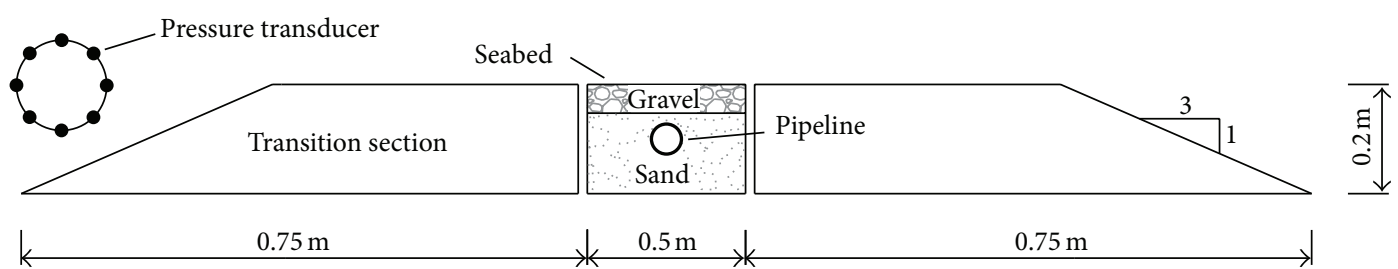

Figure 3: Test section.

TABle 1: Properties of test sand.

\begin{tabular}{lc}
\hline Properties & Values \\
\hline Shear modulus $G$ & $4 \times 10^{6} \mathrm{~Pa}$ \\
Poisson's ratio $v$ & 0.3 \\
Porosity $n_{s}$ & 0.69 \\
Permeability $k_{s}$ & $0.00075 \mathrm{~m} / \mathrm{s}$ \\
Grain size & $2 \mathrm{~mm}$ \\
\hline
\end{tabular}

Institute. The large wave flume in the laboratory is $175 \mathrm{~m}$ (575 ft.) long, $1.2 \mathrm{~m}$ ( $4 \mathrm{ft}$.) wide, and $1.5 \mathrm{~m}$ (15ft.) deep. The effective range of wave period is $0.5 \sim 6 \mathrm{~s}$. The wave-pushing plate adopts horizontal sliding plate, and the guide rail is straight line rolling type with the maximum amplitude of $300 \mathrm{~mm}$. The maximum velocity of the wave-pushing plate is below $0.75 \mathrm{~m} / \mathrm{s}$.

The experimental section for the tests on wave-soilpipeline interaction is constructed in the middle of the wave flume, as shown in Figure 2. The waves generated by the wave generator at one end of the flume spread through the test section and are absorbed by the slope beach of $3 \mathrm{H}: 1 \mathrm{~V}$ at the other end. The energy of the reflected waves is reduced to $3 \sim 20 \%$ of the incident energy.

The test section is $0.5 \mathrm{~m}$ ( $1.7 \mathrm{ft}$.) long, $0.6 \mathrm{~m}$ ( $2 \mathrm{ft}$.) wide, and $0.2 \mathrm{~m}(0.7 \mathrm{ft}$. $)$ deep. A typical cross section is shown in Figure 3 . The pipeline is buried in the sand. The upper gravel layer $\left(d_{50}=10.5 \mathrm{~mm}\right)$ with thickness of $1 \mathrm{~cm}$ is necessary to avoid the lower sand being washed away during the large wave flume tests. This layer of gravel is very permeable and may provide satisfactory pressure transmission for the sand layer. Considering the thin thickness and that the distance between the bottom of gravel layer to the center of pipeline is $9 \mathrm{~cm}$, the properties of the gravel layer could be ignored. The lower sand $\left(d_{50}=2 \mathrm{~mm}\right)$ is very uniform, and the properties of the test sand are given in Table 1.

A test section of stainless steel pipeline, $0.06 \mathrm{~m}(0.197 \mathrm{ft}$.) in diameter and $0.6 \mathrm{~m}$ (1.967 ft.) in length, and the cylindrical

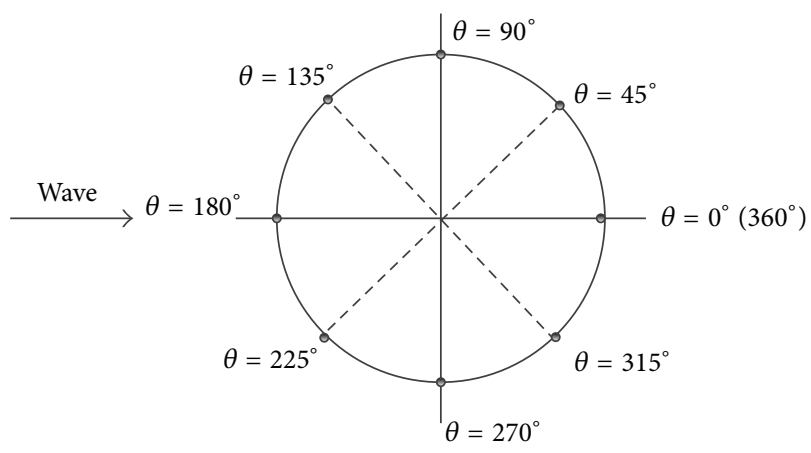

FIGURE 4: Layout of sensors.

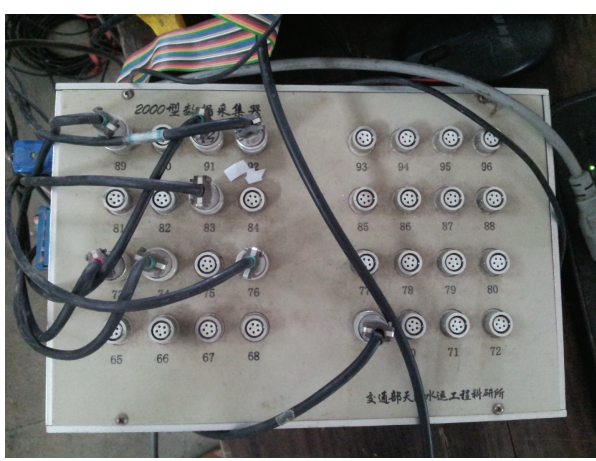

FIGURE 5: Data acquisition instrument.

surface is instrumented with eight pressure transducers. These instruments are mounted in pairs at the octant around the cylinder, as shown in Figure 4.

The 2000-type data acquisition equipment with precision of $0.01 \mathrm{kPa}$ can simultaneously acquire 64-channel data, and it is developed by Tianjin Research Institute for Water Transport Engineering of China. The synchronous acquisition of pressure sensors is ensured, as shown in Figure 5. 

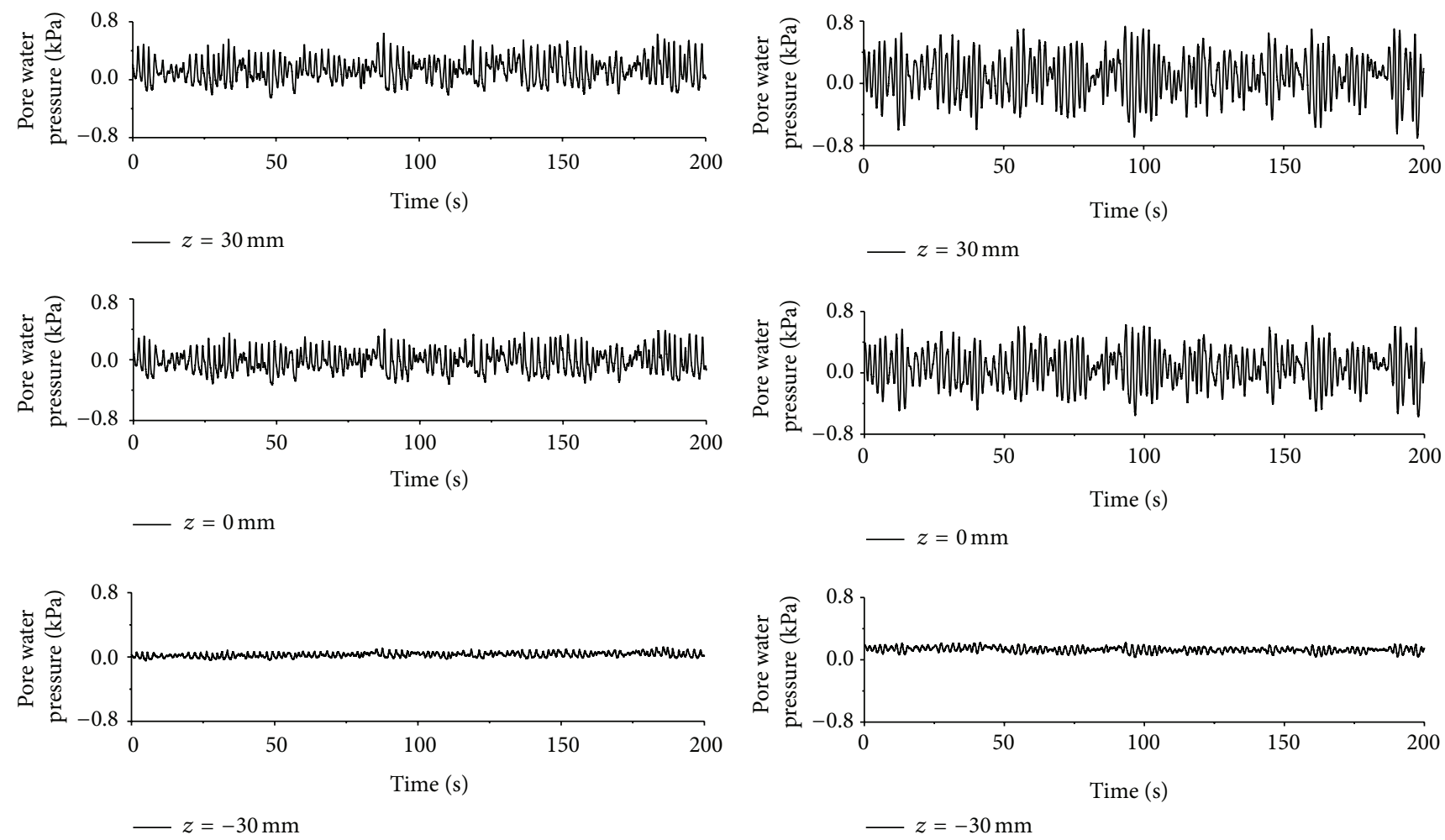

(a) $d=0.25 \mathrm{~m}$

(b) $d=0.5 \mathrm{~m}$

FIGURE 6: Measured pore pressure in seabed.

TABLE 2: Wave conditions.

\begin{tabular}{lcc}
\hline $\begin{array}{l}\text { Depth of still water } \\
d / \mathrm{m}\end{array}$ & $\begin{array}{c}\text { Significant wave } \\
\text { height } H / \mathrm{m}\end{array}$ & $\begin{array}{c}\text { Effective wave } \\
\text { period } T / \mathrm{s}\end{array}$ \\
\hline 0.15 & 0.1 & 1.92 \\
0.25 & 0.15 & 1.92 \\
0.4 & 0.185 & 1.97 \\
0.5 & 0.185 & 1.97 \\
\hline
\end{tabular}

The water depth for the test section is $0.15 \sim 0.5 \mathrm{~m}$, and the thickness of the model seabed is $h=0.15 \mathrm{~m}$. The loading waves are irregular with height $0.1 \sim 0.23 \mathrm{~m}$, and its period is $1 \sim 2.5 \mathrm{~s}$. The wave conditions are shown in Table 2. According to the research findings of Le Méhauté, the wave conditions in the tests all satisfy the cnoidal wave theory.

5.2. Test Results. The response of pore water pressure in the seabed around the pipeline during tests is shown in Figure 6. It can be found that, under the same wave conditions, the pore water pressure increases gradually with the increase of the water depth; the pore water pressure at the upper half of the seabed caused by waves greatly fluctuates, and the amplitude at the lower half remains fundamentally stable. For the sandy seabed, the excess pore pressure changes with time at the same depth, and its amplitude is different under the action of irregular waves with different wave periods. It has a large fluctuation, as shown in Figure 7, and its variation is

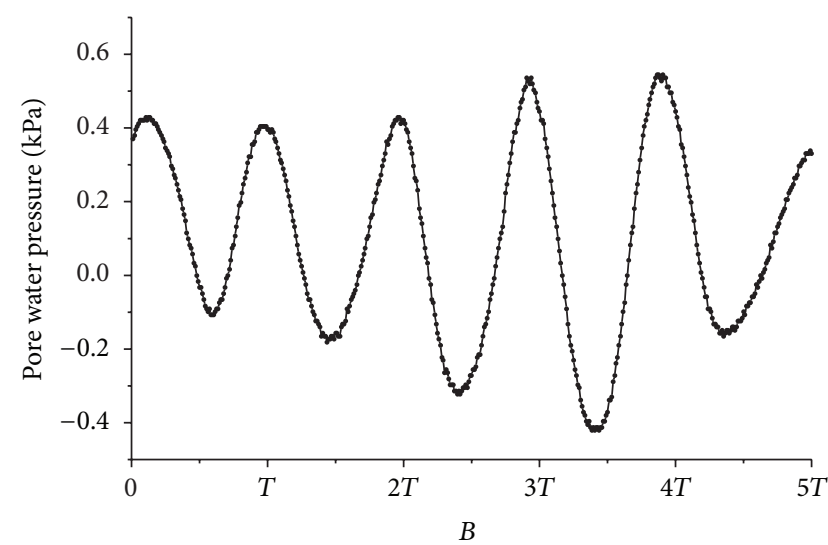

FigURE 7: Measured pore pressure under different wave periods.

different from that of the pore pressure under the action of regular waves in sandy seabed. Although the waves showed in Figure 6 belong to irregular wave, according to its wave property, it belongs to the research field of cnoidal wave.

The dynamic response of elastic seabed around the pipelines under the influence of nonlinear wave loads is studied by using the method of separation of variables. The theoretical results are compared with the measured ones in Figure 8 . The wave pressure on the surface of seabed $\left(P_{0}, z\right.$ $=0 \mathrm{~m}$ ) adopts the cnoidal nonlinear wave theory. From this figure, it can be found that the pore water pressure around 


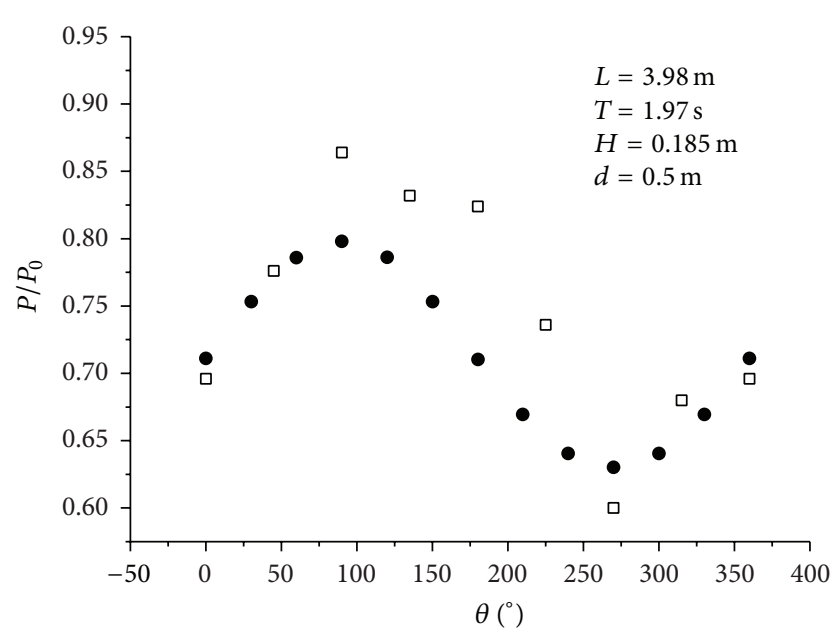

- Measurements

- Theory

(a)

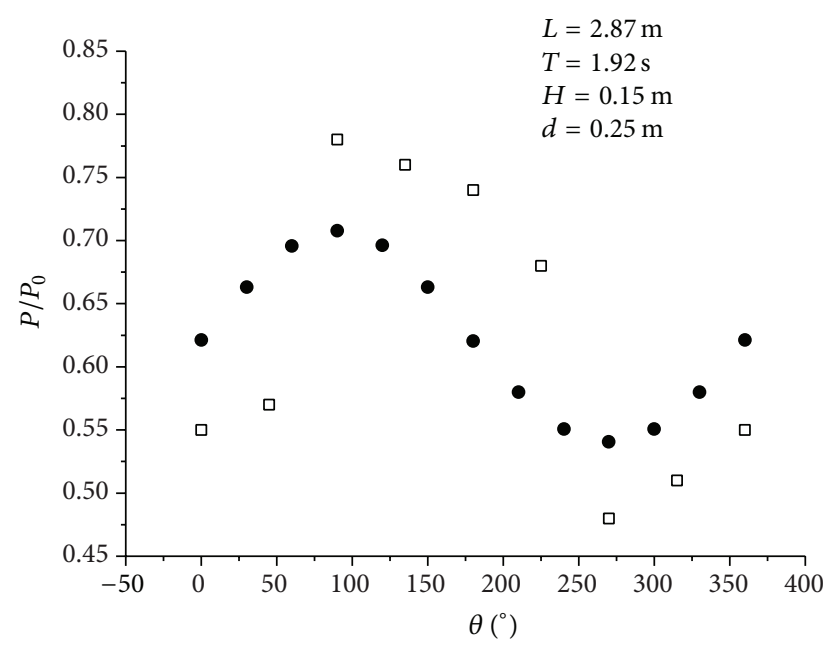

口 Measurements

- Theory

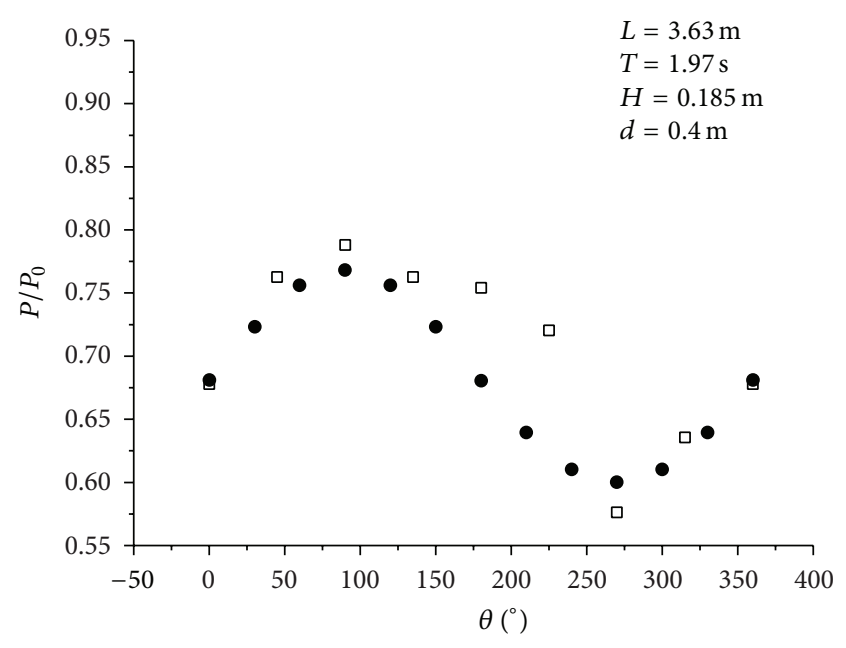

- Measurements

- Theory

(b)

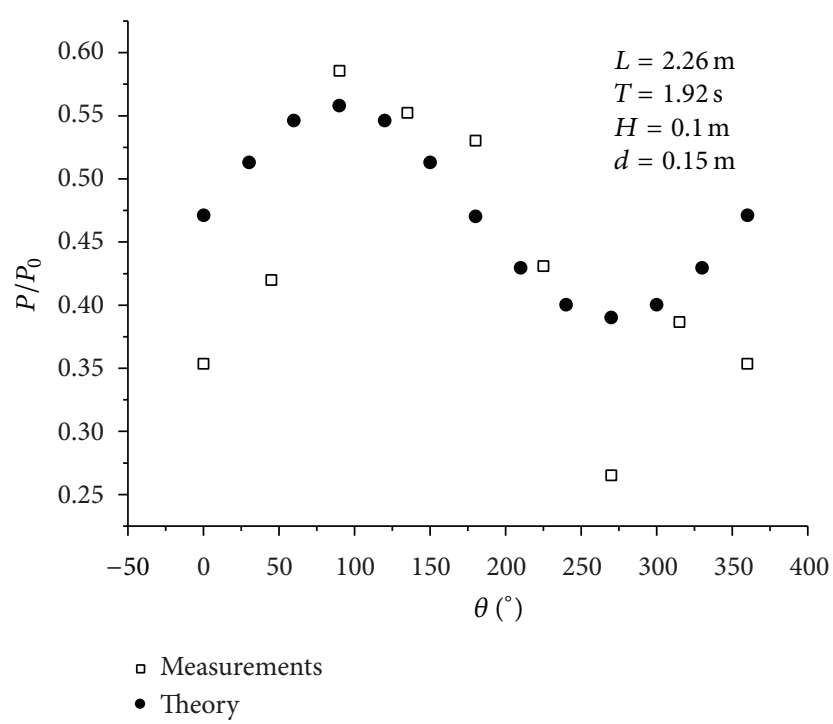

(d)

FIgURE 8: Comparison between theoretical and test results.

the pipeline has a sinusoidal distribution. The largest dynamic pore pressure is at the top of the cylinder and the smallest one exists at the bottom, and it becomes more obvious as the water depth increases. The theoretical values agree well with the test ones, and the test values of pore pressure in seabed around the pipelines are less than the theoretical ones. The theory is suitable for the infinitely deep soils, while in the tests the soils are shallow to intermediate with respect to the wavelength. The infinitely deep soils cause greater dynamic pressure attenuation than the finite depth soils; consequently, the theoretical values for the infinitely deep soils should fall below the experimental observations. At the same time, in order to prevent the sand from flowing into the sensors and influencing the test results, geotextile is packaged at the outside of the sensors during the tests, which will also make the measured results small to certain extent.

\section{Conclusions}

Based on the Biot consolidation theory and the first-order approximate cnoidal wave theory, the analytical solutions of pore water pressure around the buried pipelines in seabed with infinite depth are derived. Large wave flume tests are carried out to study the dynamic response around the pipeline under the action of nonlinear waves. The test results show that the pore water pressure in seabed around the pipeline has a sinusoidal distribution under the action of 
nonlinear waves, and the largest value occurs at the position of $90^{\circ}$ around the pipeline and the minimum one exists at the position of $270^{\circ}$. A certain point of pore water pressure in seabed has an irregularly periodical change. As the water depth increases, the pore water pressure in the seabed soils increases, and when it closes to the seabed surface, influence caused by waves will become more obvious. In this paper, the theoretical results are roughly the same as the test ones. Accordingly it can be considered that the proposed theory is feasible and valuable for marine projects.

\section{Conflict of Interests}

The authors declare that there is no conflict of interests regarding the publication of this paper.

\section{References}

[1] J. A. Putnam, "Loss of wave energy due to percolation in a permeable sea bottom," Transactions, American Geophysical Union, vol. 30, no. 3, pp. 349-356, 1949.

[2] R. O. Reid and K. Kajiura, "On the damping of gravity waves over a permeable sea bed," Transactions, American Geophysical Union, vol. 38, no. 5, pp. 662-666, 1957.

[3] P. L. F. Liu, "Damping of water waves over porous bed," Journal of the Hydraulics Division, vol. 99, no. 12, pp. 2263-2271, 1973.

[4] S. R. Massel, "Gravity waves propagated over permeable bottom," Journal of Waterway, Port, Coastal, and Ocean Engineering, vol. 102, no. 2, pp. 111-121, 1976.

[5] J. F. A. Sleath, "Wave-induced pressures in beds of sand," Journal of the Hydraulics Division, vol. 96, no. 2, pp. 367-378, 1970.

[6] H. Nakamura, R. Onishi, and H. Minamide, "On the seepage in the seabed due to waves," in Proceedings of the 20th Coastal Engineering Conference, pp. 421-428, JSCE, Taipei, Taiwan, 1973.

[7] H. Moshagen and A. Torum, "Wave induced pressures in permeable seabeds," Journal of the Waterways, Harbors and Coastal Engineering Division, vol. 101, no. 1, pp. 49-57, 1975.

[8] H. J. Prevost, O. Eide, and K. H. Anderson, "Discussion of wave induced pressure in permeable seabed," Journal of Waterways, Harbor and Coastal Engineering Division, vol. 101, pp. 464-465, 1975.

[9] O. S. Madsen, "Wave-induced pore pressure and effective stresses in a porous bed," Geotechnique, vol. 28, no. 4, pp. 377393, 1978.

[10] T. Yamamoto and Y. Suzuki, "Stability analysis of seafloor foundations," in Proceedings of the Coastal Engineering Conference, pp. 1799-1818, Sydney, Australia, 1980.

[11] T. Yamamoto, "Wave-induced pore pressures and effective stresses in inhomogeneous seabed foundations," Ocean Engineering, vol. 8, no. 1, pp. 1-16, 1981.

[12] J. R. C. Hsu, D. S. Jeng, and C. E. Lee, "Oscillatory soil response and liquefaction in an unsaturated layered seabed," International Journal for Numerical and Analytical Methods in Geomechanics, vol. 19, no. 12, pp. 825-849, 1995.

[13] H. Macpherson, "Wave forces on pipeline buried in permeable seabed," Journal of Waterway, Port, Coastal, and Ocean Engineering, vol. 104, no. 4, pp. 407-419, 1978.

[14] W. G. McDougal, S. H. Davidson, P. L. Monkmeyer, and C. K. Sollitt, "Wave-induced forces on buried pipelines," Journal of
Waterway, Port, Coastal and Ocean Engineering, vol. 114, no. 2, pp. 220-236, 1988.

[15] P. L. Monkmeyer, P. F. Mantovani, and H. Vincent, "Waveinduced seepage effects on a buried pipeline," in Proceedings of the Coastal Structures, pp. 519-531, Arlington, Tex, USA, 1983.

[16] N. W. Lai, F. R. Dominguez, and W. A. Dunlap, Numerical Solution for Determining Wave-induced Pressure Distribution around Buried Pipelines, Texas A\&M University, College Station, Tex, USA, 1974.

[17] G. P. Lennon, "Wave-induced forces on buried pipelines," in Proceedings of the Coastal Structures, pp. 505-518, Arlington, Tex, USA, March 1983.

[18] P. L. F. Liu and T. P. O’Donnell, "Wave-induced forces on buried pipelines in permeable seabed," in Proceedings of the Civil Engineering in the Oceans IV Conference, pp. 111-121, San Francisco, Calif, USA, September 1979.

[19] A. H.-D. Cheng and P. L.-F. Liu, "Seepage force on a pipeline buried in a poroelastic seabed under wave loadings," Applied Ocean Research, vol. 8, no. 1, pp. 22-32, 1986.

[20] W. Magda, "Wave-induced uplift force acting on a submarine buried pipeline: finite element formulation and verification of computations," Computers and Geotechnics, vol. 19, no. 1, pp. 4773, 1996.

[21] W. Magda, "Wave-induced uplift force on a submarine pipeline buried in a compressible seabed," Ocean Engineering, vol. 24, no. 6, pp. 551-576, 1997.

[22] W. Magda, "Wave-induced cyclic pore-pressure perturbation effects in hydrodynamic uplift force acting on submarine pipeline buried in seabed sediments," Coastal Engineering, vol. 39, no. 2-4, pp. 243-272, 2000.

[23] D.-S. Jeng and L. Cheng, "Wave-induced seabed instability around a buried pipeline in a poro-elastic seabed," Ocean Engineering, vol. 27, no. 2, pp. 127-146, 2000.

[24] D. S. Jeng and Y. S. Lin, "Wave-induced pore pressure around a buried pipeline in gibson soil: finite element analysis," International Journal for Numerical and Analytical Methods in Geomechanics, vol. 23, no. 13, pp. 1559-1578, 1999.

[25] D. S. Jeng and Y. S. Lin, "Response of inhomogeneous seabed around buried pipeline under ocean waves," Journal of Engineering Mechanics, vol. 126, no. 4, pp. 321-332, 2000.

[26] D. S. Jeng, "Numerical modeling for wave-seabed-pipe interaction in a non-homogeneous porous seabed," Soil Dynamics and Earthquake Engineering, vol. 21, no. 8, pp. 699-712, 2001.

[27] S. L. Dunn, P. L. Vun, A. H. C. Chan, and J. S. Damgaard, "Numerical modeling of wave-induced liquefaction around pipelines," Journal of Waterway, Port, Coastal \& Ocean Engineering, vol. 132, no. 4, pp. 276-288, 2006.

[28] F. P. Gao, D. S. Jeng, and H. Sekiguchi, "Numerical study on the interaction between non-linear wave, buried pipeline and nonhomogenous porous seabed," Computers and Geotechnics, vol. 30, no. 6, pp. 535-547, 2003.

[29] L.-Z. Wang, D.-Z. Pan, and C.-H. Pan, "Analytical solution for wave-induced response of seabed with variable shear modulus," China Ocean Engineering, vol. 21, no. 3, pp. 389-400, 2007.

[30] M.-T. Luan, P. Qu, and Q. Yang, "Nonlinear wave-induced transient response of pipeline-seabed interaction," Rock and Soil Mechanics, vol. 28, supplement 1, pp. 709-714, 2007 (Chinese).

[31] B. Le Méhauté, An Introduction to Hydrodynamics and Water Waves, Springer, New York, NY, USA, 1976.

[32] D. Qiu, Waves and Seepage Mechanics, National Defense Publishing House, Beijing, China, 2006. 
[33] P. J. Lynett, P. L.-F. Liu, I. J. Losada, and C. Vidal, "Solitary wave interaction with porous breakwaters," Journal of Waterway, Port, Coastal and Ocean Engineering, vol. 126, no. 6, pp. 314-322, 2000.

[34] S. Song, Y.-X. You, and G. Wei, "The interaction of the solitary wave with a vertically walled porous structure," Ocean Engineering, vol. 25, no. 4, pp. 7-14, 2007 (Chinese).

[35] Q. Li, H. Huang, and J.-M. Zhan, "Solitary wave-induced seepage effects on the bottom of vertical cylinder resting on porous seabed," Journal of Tropical Oceanography, no. 5, pp. 4247, 2013 (Chinese).

[36] H. Xiao and W. Huang, "Three-dimensional numerical modeling of solitary wave breaking and force on a cylinder pile in a coastal surf zone," Journal of Engineering Mechanics, vol. 141, no. 8, Article ID A4014001, 2015.

[37] Z.-C. Sun and D.-H. Qiu, "Nonlinear wave force on a buried pipeline in shallow water," Journal of Dalian University of Technology, supplement 1, pp. 95-98, 2000.

[38] Y.-F. Xu, X.-H. Xia, J.-H. Wang, and J.-J. Chen, "Response of seabed to cnoidal waves," Journal of Shanghai Jiaotong University, vol. 47, no. 10, pp. 1580-1584, 2013 (Chinese).

[39] X.-L. Zhou, J. Zhang, J.-H. Wang, Y.-F. Xu, and D.-S. Jeng, "Stability and liquefaction analysis of porous seabed subjected to cnoidal wave," Applied Ocean Research, vol. 48, pp. 250-265, 2014.

[40] M. A. Biot, "General theory of three-dimensional consolidation," Journal of Applied Physics, vol. 12, no. 2, pp. 155-164, 1941.

[41] J. Bear, Dynamics of Fluids in Porous Media, Soil Science, 1972.

[42] K. Kokkino Wrachos, "Hydrodynamic analysis of large offshore structure on porous elastic seabed," in Proceedings of the 4th International OMAE Symposium, Tokyo, Japan, 1985. 


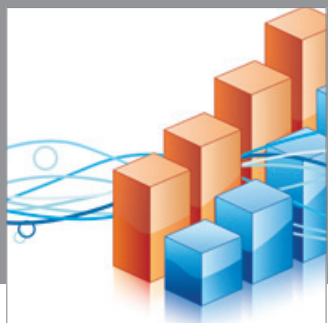

Advances in

Operations Research

mansans

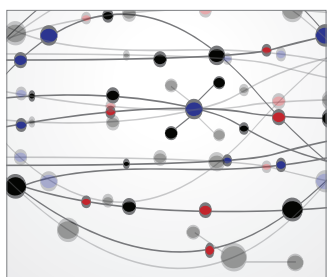

The Scientific World Journal
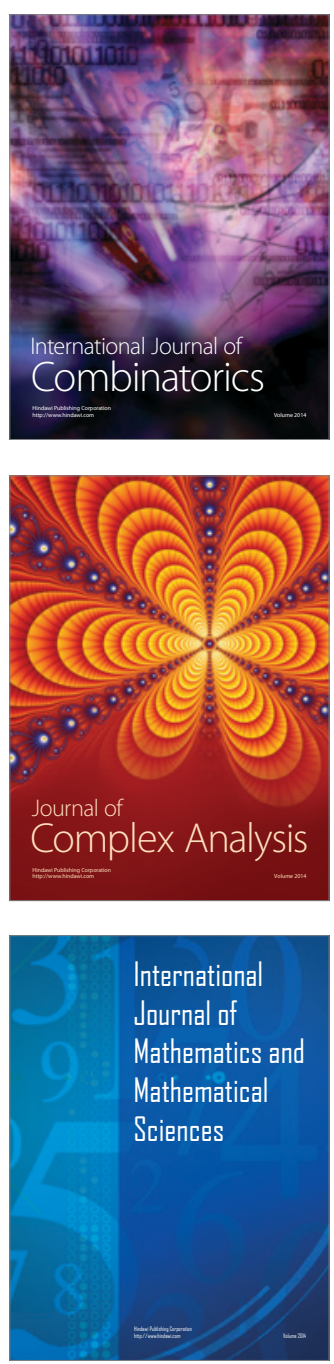
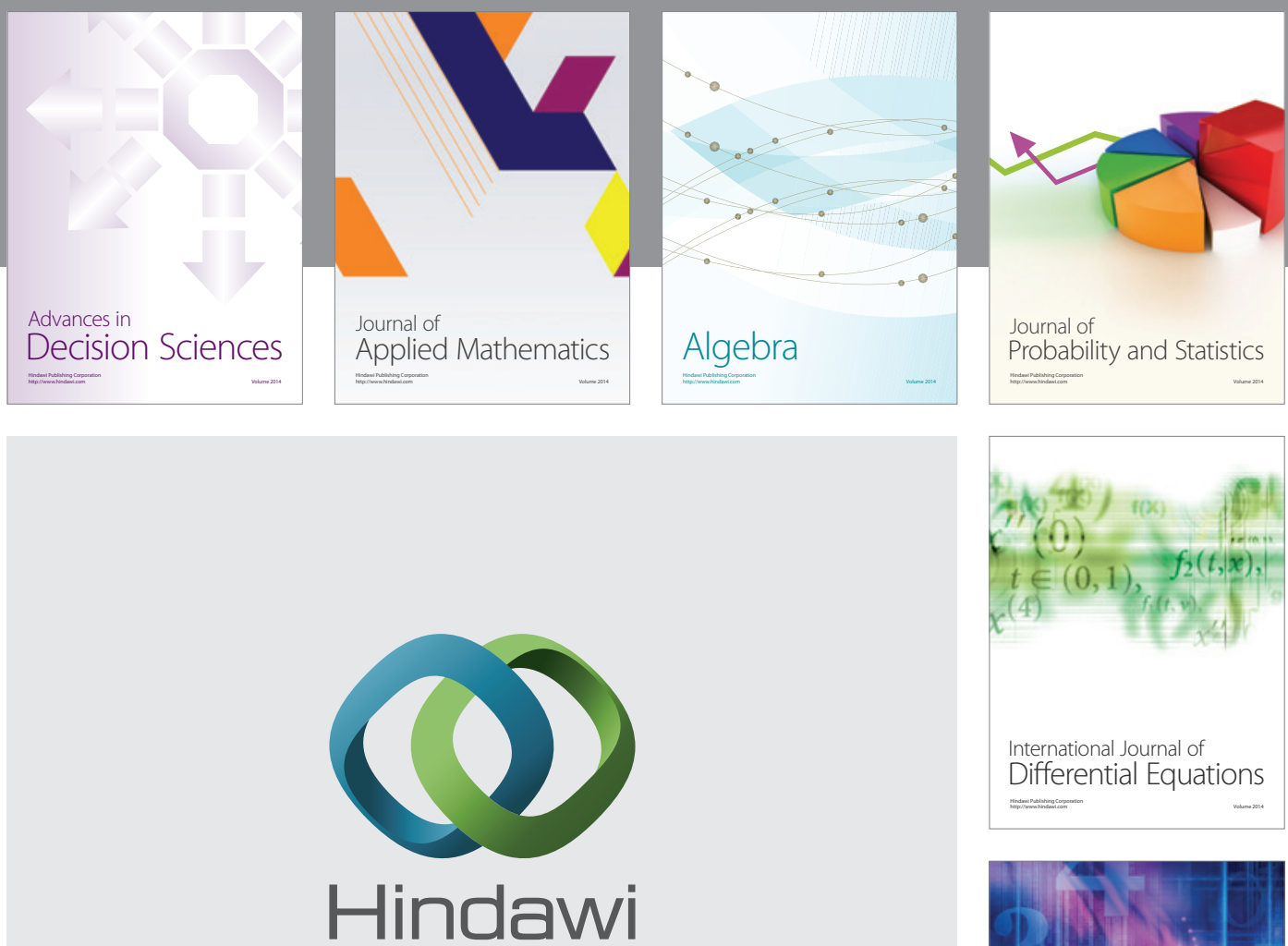

Submit your manuscripts at http://www.hindawi.com
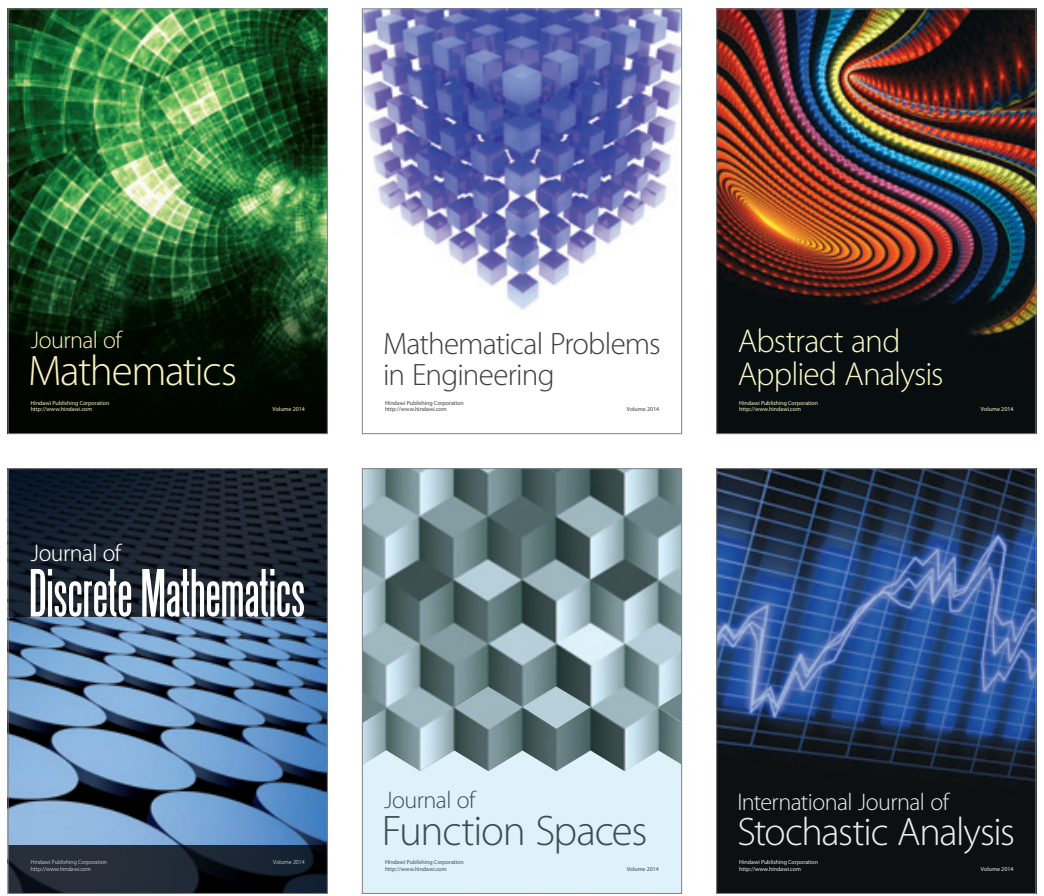

Journal of

Function Spaces

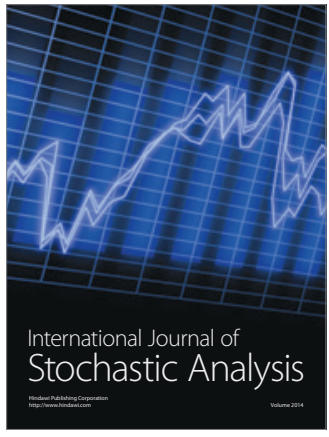

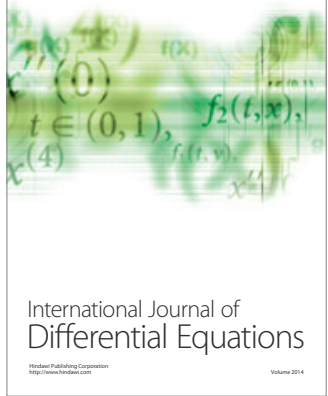
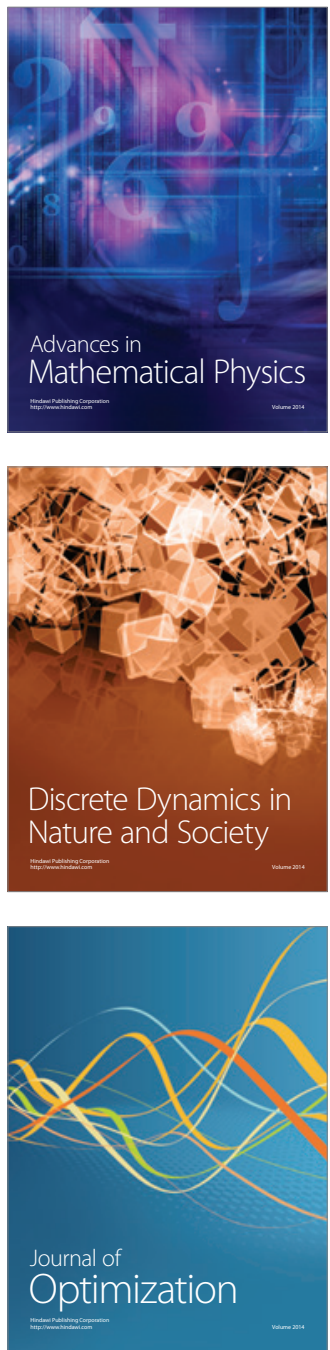